\section{SARS-CoV-2-specific IgM screening has low sensitivity for identifying potentially infectious travellers}

Sir,

During the COVID-19 pandemic, there has been substantial interest in the ability to rapidly diagnose infection serologically, in particular to clear individuals to attend their workplace or for travel. Currently, a negative test result for severe acute respiratory syndrome-coronavirus-2 (SARSCoV-2)-specific IgM immediately prior to travel has been mandated for some destinations in addition to nucleic acid testing (NAT). ${ }^{1}$ While pathogen-specific $\operatorname{IgM}$ is an early immune marker for many infectious diseases, there are exceptions. Many respiratory viruses, including SARS-CoV-2, demonstrate delayed IgM seroconversion often greater than 10 days from onset of illness, and this follows the appearance of specific IgG antibodies. ${ }^{2}$ We explored the utility of SARS-CoV-2-specific IgM test as a marker of infective risk and thus as a guide for international traveller screening.

We performed a retrospective cross-sectional study on all SARS-CoV-2-specific serology performed by NSW Health Pathology's Institute of Clinical Pathology and Medical Research, Australia before 10 November 2020. Sera were tested on a validated in-house quantitative indirect immunofluorescence assay (IFA) for SARS-CoV-2-specific IgM, $\operatorname{IgA}$ and $\operatorname{IgG}$ detection with a sensitivity of $91.3 \%$ and specificity of $98.9 \%$ for any antibody class and a sensitivity of $62.2 \%$ and specificity of $99.7 \%$ for IgM for NAT-positive patients. ${ }^{3}$ Clinical information was obtained from pathology request forms.

Individuals were classified as infectious if they had confirmed or probable SARS-CoV-2 infection (using Australian Public Health definitions) ${ }^{4}$ and were within 14 days from illness onset (the estimated maximum duration of infectivity adopted by the Communicable Diseases Network of Australia in their guidance on release from isolation). ${ }^{4} \mathrm{~A}$ confirmed case was defined by laboratory confirmation using a positive SARS-CoV-2 NAT, isolation of SARS-CoV-2 in cell culture or SARS-CoV-2-specific IgG seroconversion or a four-fold increase in $\mathrm{IgG}$ titres. A probable case was defined as someone who had a positive SARS-CoV-2-specific IgG with either clinical or epidemiological history compatible with SARS-CoV-2 infection. ${ }^{4}$

Individuals with confirmed or probable infection were considered non-infectious once they were $>14$ days after illness onset, as were individuals in whom SARS-CoV-2 infection was excluded with a negative NAT. As the current study aimed to determine the utility of $\operatorname{IgM}$ as a screening test prior to travel and the timing of IgM positivity relative to infective course could not be determined, individuals with confirmed or probable infection in whom the date of illness onset was unknown, or who were asymptomatic, were not included in the evaluation. Similarly, as the COVID-19 status could not be confirmed, individuals with a positive SARSCoV-2-specific IgG but not meeting public health definitions for a confirmed or probable infection were excluded. Several individuals had multiple serology tests at different time points in their illness and these were assessed individually. The sensitivity, specificity, positive predictive value and negative predictive value of a positive SARS-CoV-2-specific IgM being able to identify an infectious individual were calculated.

Ethics was approved by Western Sydney Local Health District Human Research Ethics Committee (2008-10 QA).

The study included 7075 individuals, after excluding confirmed and probable cases without a known illness onset date $(n=419)$ and cases with positive SARS-CoV-2-specific IgG who did not meet the criteria for confirmed or probable infection ( $n=5157)$ (Fig. 1). In total, 420 samples were taken while an individual was infectious and 7824 were taken while

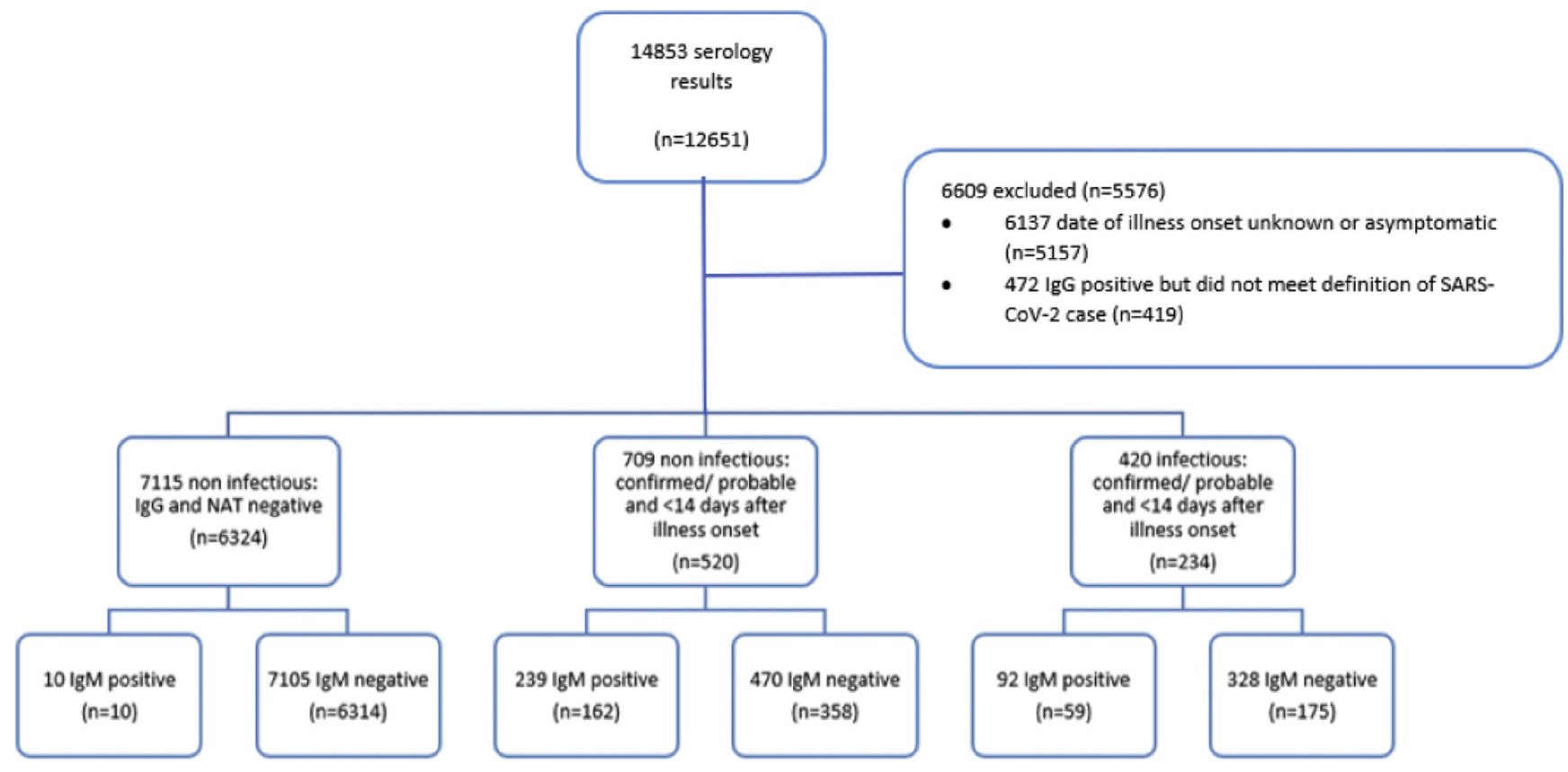

Fig. 1 Breakdown of serology results by IgM results and infectiousness. 
Table 1 IgM detection between infectious and non-infectious periods

\begin{tabular}{lccc}
\hline & Infectious & Non-infectious & Total \\
\hline IGM positive & 92 & 249 & 341 \\
IGM negative & 328 & 7575 & 7903 \\
Total & 420 & 7824 & 8244 \\
& & & \\
\hline
\end{tabular}

an individual was non-infectious. SARS-CoV-2-specific IgM results by infectious or non-infectious status are indicated in Table 1. Mean SARS-CoV-2-specific IgM titres peaked in the third week and were undetectable by approximately 10 weeks, on average.

The specificity of SARS-CoV-2-specific IgM detection within the presumed 14-day infectious period was $96.8 \%$ and sensitivity was $21.9 \%$. Figure 2 summarises the positive and negative predictive value over a range of prevalence. At the end of December 2020 there were only 220 active SARS-CoV-2 infections in Australia. ${ }^{5}$ Assuming an estimated infection to case ratio of $3.5,{ }^{6}$ this would suggest a prevalence of infectious individuals in Australia (population 25 million) of $0.003 \%$. At this prevalence, the positive predictive value of $\operatorname{IgM}$ to predict an individual is infectious would be $0.007 \%$. Even at the peak of the second wave in Australia, when there were 8195 active cases on 12 August 2020, the positive predictive value of IgM to determine infectious state would be only $0.07 \%$.

While serology is important in understanding the extent of antibody response and sero-prevalence, its role in diagnosis of infection in the acute, infectious period remains limited. This brief review reinforces that serology, in particular SARS-CoV-2-specific IgM, is not useful in identifying infectious individuals. This has implications for the use of serology in determining suitability for travel.

This study demonstrates very low positive predictive value for a positive SARS-CoV-2-specific IgM to potentially identify an infectious individual. In Australia, where the prevalence of infection is low $(<1 \%),{ }^{6}$ the positive predictive value of a SARS-CoV-2-specific IgM to detect an infectious individual is less than $1 \%$ and a positive result may exclude an individual who may have already recovered from travelling. There is evidence that development of antibodies to SARS-CoV-2 may directly coincide with a loss of infectivity. ${ }^{7}$ Even in a high prevalence setting, neither a negative nor a positive SARS-CoV-2-specific IgM would be able to appropriately exclude infectious individuals from travelling. The variable accuracy of serological testing using various commercial and rapid SARS-CoV-2-specific IgM, which have differing sensitivity for $\operatorname{IgM}{ }^{8}$ further makes interpretation difficult, particularly in the context of a negative SARS-CoV-2 NAT. A study looking at different commercial SARS-CoV-2 assays found the specificity of IgM to be high (96.3-99.7\%), however the sensitivity varied between assays $(42.0-82.7 \%){ }^{8}$ Assays with a lower sensitivity would perform poorer in predicting the infectivity of an infectious traveller.

Asymptomatic individuals may be less likely to develop SARS-CoV-2-specific IgM, ${ }^{9}$ although asymptomatic individuals can pose a risk of transmission, ${ }^{10}$ which further reduces the utility of SARS-CoV-2-specific IgM for pretravel screening. The rollout of COVID-19 vaccines will also impact the utility of SARS-CoV-2-specific IgM to identify infectious individuals, depending on administered vaccine components and the antigens utilised in serological assays; in particular, the COVID-19 vaccine can elicit an IgM response in a subset of individuals. ${ }^{11}$ In one study, all participants demonstrated an IgM response after receiving the second but not first dose of the BNT162b2 mRNA COVID19 vaccine. $^{12}$

In conclusion, SARS-CoV-2-specific IgM measurement is not a useful screening test as an addition to NAT to identify infectious individuals prior to travel.

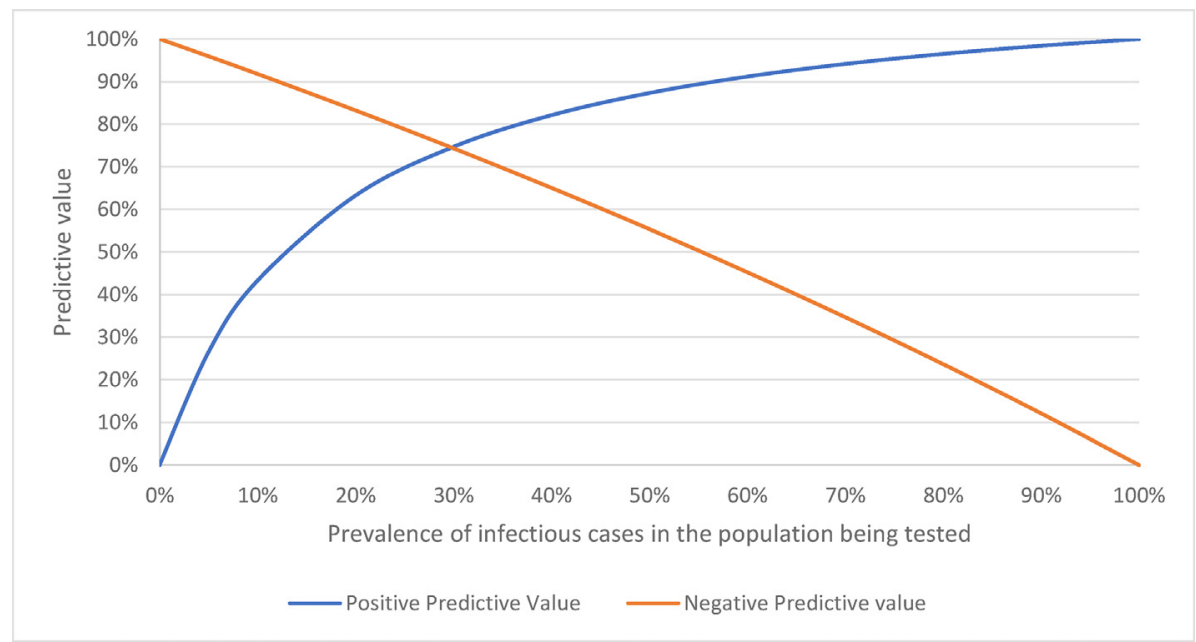

Fig. 2 Change in positive and negative predictive value according to prevalence. 
Tasnim Hasan ${ }^{1}$, H. Ling Lim ${ }^{1}$, Linda Hueston ${ }^{1}$, Dominic E. Dwyer ${ }^{1,2,3}$, Matthew O'Sullivan ${ }^{1,2,3}$

${ }^{1}$ Centre for Infectious Diseases and Microbiology Laboratory Services, NSW Health Pathology - Institute of Clinical Pathology and Medical Research, Westmead Hospital, Westmead, NSW, Australia; ${ }^{2}$ Centre for Infectious Diseases and Microbiology - Public Health, Westmead Hospital, Westmead, NSW, Australia; ${ }^{3}$ Marie Bashir Institute for Infectious Diseases and Biosecurity, Sydney Medical School, The University of Sydney, NSW, Australia

Contact Dr Tasnim Hasan.

E-mail: Tasnim.hasan@health.nsw.gov.au

1. Embassy of the People's Republic of China in the United States of America. Notice on the requirement adjustment of the nucleic acid rtPCR and IgM serum antibody tests. Dec 2020; cited Jul 2021. www. china-embassy.org/eng/notices/t1841416.htm

2. Hsueh PR, Huang LM, Chen PJ, et al. Chronological evolution of IgM, $\operatorname{Ig} \mathrm{A}, \operatorname{IgG}$ and neutralisation antibodies after infection with SARSassociated coronavirus. Clin Microbiol Infect 2004; 10: 1062-6.

3. Hueston L, Kok J, Guibone A, et al. The antibody response to SARSCoV-2 infection. Open Forum Infect Dis 2020; 7: ofaa387.

4. Australian Government Department of Health. Coronavirus disease 2019 (COVID-19): CDNA National guidelines for public health units. Cited Jul 2021. https://www1.health.gov.au/internet/main/publishing. nsf/Content/cdna-song-novel-coronavirus.htm

5. Live COVID. Daily active cases. Nov 2020; cited Jul 2021. https:// covidlive.com.au/report/daily-active-cases/aus

6. Gidding H, Machalek D, Hendry A, et al. Seroprevalence of SARSCoV-2-specific antibodies in Sydney, Australia following the first epidemic wave in 2020. Med J Aust 2021; 214: 179-85.

7. Kwon JS, Kim JY, Kim MC, et al. Factors of severity in patients with COVID-19: cytokine/chemokine concentrations, viral load, and antibody responses. Am J Trop Med Hyg 2020; 103: 2412-8.

8. Harritsh $\varnothing \mathrm{j}$ LH, Gybel-Brask M, Afzal S, et al. Comparison of 16 serological SARS-CoV-2 immunoassays in 16 clinical laboratories. J Clin Microbiol 2021; 59: e02596-20.

9. Shirin T, Bhuiyan TR, Charles RC, et al. Antibody responses after COVID-19 infection in patients who are mildly symptomatic of asymptomatic in Bangladesh. Int J Infect Dis 2020; 101: 220-5.

10. Letizia AG, Ramos I, Obla A, et al. SARS-CoV-2 transmission among marine recruits during quarantine. N Engl J Med 2020; 383 2407-16.

11. Jalkanen P, Kolehmainen P, Häkkinen HK, et al. COVID-19 mRNA vaccine induced antibody responses against three SARS-CoV-2 variants. Nat Commun 2021; 12: 3991.

12. Izumo $\mathrm{T}$, Kuse $\mathrm{N}$, Awano $\mathrm{N}$, et al. Side effects and antibody titer transition of the BNT162b2 messenger ribonucleic acid coronavirus disease 2019 vaccine in Japan. Respir Investig 2021; 59: 635-42.

DOI: https://doi.org/10.1016/j.pathol.2021.08.002

\section{Verification of the BioFire FilmArray Pneumonia Plus Panel for pathogen screening of respiratory specimens}

Sir,

Pneumonia-like illnesses cause significant morbidity and mortality in the hospital and community setting. ${ }^{1}$ The most common aetiological agents, bacteria (intracellular or extracellular) and viruses, often exhibit similar clinical presentations. Empiric broad spectrum antimicrobial therapy is recommended for suspected bacterial infections; however, it is unnecessary in many cases where the aetiological agent is proven to be viral, contributing to its overuse. Rapid laboratory diagnostic testing, as such, forms a critical component of the clinical response to lower respiratory tract infections (LRTIs), as well as an effective antimicrobial stewardship strategy. Pathogen identification by culture-based methods and antimicrobial susceptibility testing can take 48-72 hours. $^{2}$ Ancillary specific rapid diagnostic methods have been developed for some of the most common aetiological agents of LRTIs; however, these may be ineffective in diagnosing infections with a complex aetiology.

Rapid multiplex polymerase chain reaction (PCR) panels that detect a range of aetiological agents and the most common antimicrobial resistance targets have emerged to meet this clinical gap. ${ }^{3}$ One system that has recently been approved is the BioFire FilmArray Pneumonia Plus (PNplus) Panel (bioMérieux, France). This assay incorporates automated testing for 18 bacteria (11 Gram-negative, 4 Grampositive, 3 atypical) and nine viruses that cause LRTIs as well as seven common antibiotic resistance markers. ${ }^{4}$ Validation studies in various settings are emerging, revealing high sensitivity (91-98\%) and specificity (76-87\%) for targets available for testing. 5,6

This study aimed to assess the accuracy of the PNplus as part of a verification process at the Nepean Microbiology Laboratory, Penrith, Australia, which provides microbiology diagnostic services to the Nepean Blue Mountains Local Health District. Sputum and bronchoalveolar (BAL) samples were selected from inpatients who had organisms or antimicrobial resistance genes of interest identified by National Associating of Testing Authorities Australia (NATA) approved conventional techniques. In the case of rarely encountered viral and atypical targets, previously stored sputum and BAL frozen specimens in normal saline were used. For rarely encountered bacterial and antimicrobial resistance targets, a single colony of interest was inoculated into an inpatient specimen which had been processed via conventional methods and had no pathogens isolated.

Sputum and BAL samples from hospital inpatients were processed ideally within 2 hours of collection. Samples were inoculated onto $5 \%$ horse blood agar, chocolate agar, Brilliance UTI Clarity agar (ThermoScientific, Australia) and incubated at $37^{\circ} \mathrm{C}$ on $5 \% \mathrm{CO}_{2}$. Plates were examined at 24 and 48 hours for bacterial growth. Predominant pathogens were searched for and identified using matrix assisted laser desorption ionisation-time of flight mass spectrometry (MALDI-TOF MS) (Bruker Daltonics, Germany). Results were considered negative if there was no significant growth or a non-pathogenic normal respiratory flora was isolated. Susceptibility testing was performed by VITEK 2 (bioMérieux, France) and/or disk diffusion methods as dictated by the Clinical and Laboratory Standards Institute (CLSI) guidelines. Etest (bioMérieux, France) was used as a substitute for broth microdilution and CLSI guidelines were used to interpret results. Phenotypic susceptibility patterns were reviewed, and if consistent with multi-drug resistance, cultured isolates underwent additional testing using inhouse PCRs performed on the Rotor-Gene 3000 or RotorGene Q (Qiagen, Germany). All Staphylococcus were PCR tested for the presence of mec and nuc genes using primers and probes adapted from previous reports. ${ }^{7,8} \mathrm{CTX}$ $\mathrm{M}$ and IMP were detected in Enterobacteriaceae using an assay adapted from previous reports. ${ }^{9,10}$ The GeneXpert Carba-R (Cepheid, USA) was used for detection of NDM. 